\title{
Skin Diseases and Allergy-Diencephalic Irradiation
}

U.

\author{
Granelli
}

Hospital «San Gallicano», Roma (Italy)

Author's address: Dr. U. Granelli, Hospital «San Gallicano», Roma (Italy)

Most skin diseases may be considered as cutaneous reactions in which an allergy dominates.

Daily life offers a great number of occasions in which patients come in contact with substances

with sensitizing actions. In other cases, the cutaneous disease may be of microbic or mycotic origin but with an allergic mechanism.

Studies of the relation between neuropsychic factors and allergy shows that alterations in the hypothalamic centers represent the centre of the sensitization. In numerous cases of eczema, dien-cephalic irradiation produces an anti-allergic effect, with consistent and progressive subjective and objective improvement in a short time. 